$$
\text { "tmcs-horvath" — 2013/5/26 - 0:05 — page } 3 \text { - \#1 }
$$

\title{
Mathematics in Good Will Hunting II: Problems from the students perspective
}

\author{
Gábor Horváth, József Korándi and Csaba Szabó
}

\begin{abstract}
This is the second part of a three paper long series exploring the role of mathematicians and of the mathematical content occurring in popular media. In particular we analyze the drama film Good Will Hunting. Here we investigate the mathematical content of the movie by considering the problems appearing in it. We examine how a mathematician or a mathematics student would solve these problems. Moreover, we review how these problems could be integrated into the higher education of Hungary.
\end{abstract}

Key words and phrases: mathematics in media, Good Will Hunting, popularize mathematics.

ZDM Subject Classification: D55, C35, K35.

\section{Introduction}

We continue our series of 3 papers, started by [6]. In this paper we analyze the mathematics appearing in Good Will Hunting. In Section 2 we explain the central mathematical problem of the movie. It will be clearly seen, that both the problem and the solution is well-considered and purposeful. The solution is complex, but the result can be presented both in a brief or in a complicated way. In Section 3 we consider very briefly what is written on the board when professor Lambeau addressed the mass of students in the lecture hall. In Section 4 we investigate the

The work/publication is supported by the TÁMOP-4.2.2/B-10/1-2010-0024 project. The project is co-financed by the European Union and the European Social Fund.

Copyright (c) 2013 by University of Debrecen 


$$
\text { "tmcs-horvath" — 2013/5/26 - 0:05 — page } 4 \text { - \#2 }
$$

second problem, consisting of two parts, written on the board, and reveal that the solution for the second part is not complete in the film. Finally, we finish in Section 5 by investigating the problem Will Hunting and professor Lambeau solve together in Lambeau's office. Throughout the paper we carefully show what preliminary knowledge a mathematics student needs for understanding the different ideas. Then, we review what courses at the Hungarian Universities ELTE (Eötvös Loránd University), DE (University of Debrecen) and SzTE (University of Szeged) cover these prerequisites, and how the different exercises of the show can be integrated into these classes. We hope that the reader will entertain himself reading this note as much as we entertained ourselves figuring out the details of the different solutions of these problems.

\section{Pathfinding in graphs}

The following problem occurred first in the movie. It was written on the left hand side of the board as follows.

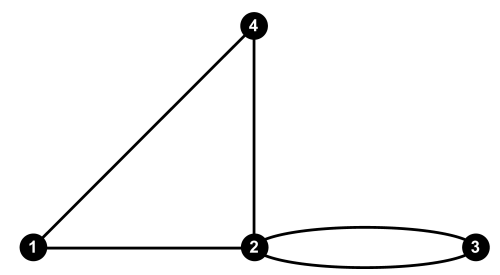

Figure 1. The graph $G$

Problem 1. $G$ is the graph on the vertex set $V=\{1,2,3,4\}$ and with edges $(1,2),(1,4),(2,3),(2,3),(2,4)((2,3)$ is a double edge $)$. Find:

(1) The adjacency matrix $A$.

(2) The matrix giving the number of 3 step walks.

(3) The generating function for walks from $i \rightarrow j$.

(4) The generating function for walks from $1 \rightarrow 3$.
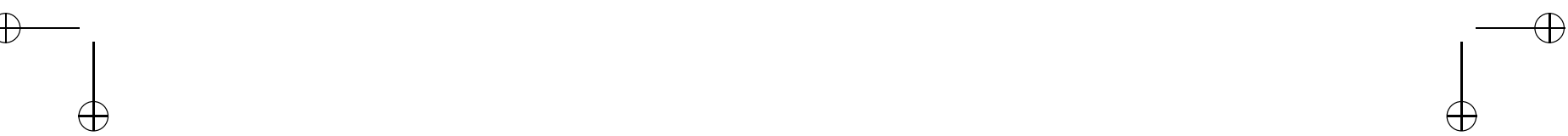


$$
\text { "tmcs-horvath" — 2013/5/26 - 0:05 — page } 5 \text { - \#3 }
$$

Will Hunting's solution. Will Hunting wrote his solution on the right hand side of the board, as follows.

Sol. 1.

$$
A=\left(\begin{array}{llll}
0 & 1 & 0 & 1 \\
1 & 0 & 2 & 1 \\
0 & 2 & 0 & 0 \\
1 & 1 & 0 & 0
\end{array}\right) .
$$

Sol. 2 .

$$
A^{3}=\left(\begin{array}{cccc}
2 & 7 & 2 & 3 \\
7 & 2 & 12 & 7 \\
2 & 12 & 0 & 2 \\
3 & 7 & 2 & 2
\end{array}\right)
$$

Sol. 3. $\quad \Gamma^{\omega}\left(p_{i} \rightarrow p_{j}, z\right)=\sum_{n=0}^{\infty} \omega_{n}(i \rightarrow j) z^{n}=\frac{\operatorname{det}\left(\mathbf{1}_{i j}-z A_{i j}\right)}{\operatorname{det}(\mathbf{1}-z A)}$

Sol. 4. $\quad\left|\begin{array}{ccc}-z & 0 & -z \\ 1 & -2 z & -z \\ -z & 0 & 1\end{array}\right| \cdot\left|\begin{array}{cccc}1 & -z & 0 & -z \\ -z & 1 & -2 z & -z \\ 0 & -2 z & 1 & 0 \\ -z & -z & 0 & 1\end{array}\right|^{-1}=$
$\quad=\frac{2 z^{3}+2 z^{2}}{4 z^{4}-2 z^{3}-7 z^{2}+1}=2 z^{2}+2 z^{3}+14 z^{4}+18 z^{5}+94 z^{6}+\ldots$

In this section we analyze and explain the mathematical impact and the solution of this problem from the perspective of a mathematician and a student with high enough mathematical background.

The proper solution cannot be explained in a few words since it uses a fair amount of the following university classes.

- Linear algebra: elementary theory of matrices, powers of matrices, Jordan normal-form.

- Analysis: convergence in normed vector spaces, power series, convergence of power series.

- Combinatorics: generating function, counting, recurrence formulae.

- Graph theory: adjacency matrix, paths, powers of the adjacency matrix. 


$$
\text { "tmcs-horvath" — 2013/5/26 - 0:05 — page } 6 \text { - \#4 }
$$

\subsection{The solution for a mathematician}

Let us consider first the impression of a mathematician. Although considered to be experienced professors of algebra and discrete mathematics, teaching both linear algebra and discrete mathematics on almost all university levels, none of the authors was aware of this issue about the generating functions for the number of paths before investigating the problem from the movie. They figured out a possible solution for this problem on their own, using the guidance of Will's answers on the board. The solution from their point of view can be interpreted in the following way.

The problem is about finding the number of walks from a vertex $i$ to a vertex $j$ in a graph $G$. From now on we use the graph theoretical notions from [8]. Let $G$ be a graph with vertex set $V=\{1,2,3,4\}$ and edge set $E=\{(1,2),(1,4),(2,4)$, $(2,3),(2,3)\}$, where $(2,3)$, is a double edge (see Figure 1$)$.

The first exercise is to find the adjacency matrix of this graph, and Sol. 1 is clearly correct. The second exercise was to provide a matrix which gives the number of 3 step walks. This matrix is $A^{3}$, and it is presented in Sol. 2.

The third question needs a bit more consideration. We know how to compute the number of paths of given length, we understand the notion of generating function of an infinite sequence, but this particular generating function is not one we usually teach. The board from the movie gives us some hint.

Let $\omega_{n}(i \rightarrow j)$ denote the number of walks of length $n$ from vertex $i$ to vertex $j$. As $A^{n}$ encodes the number of $n$ step walks from a point to another, $\omega_{n}(i \rightarrow j)$ is the $i j$ entry $\left[A^{n}\right]_{i}^{j}$ of the matrix $A^{n}$. The generating function is an analytic function defined by its power series $f_{i, j}(z)=\sum_{n=0}^{\infty} \omega_{n}(i \rightarrow j) \cdot z^{n}$, that is the coefficient of $z^{n}$ is the number of $n$ step walks from $i$ to $j$.

It does not take much of a leap to consider the matrix power series $F(z)=$ $\sum_{n=0}^{\infty} A^{n} \cdot z^{n}$. Or maybe it does. This is the key point to the solution of the problem. First, we sketch the solution, then make every step precise. Considering $F(z)$ as a formal sum, we have $[F(z)]_{i}^{j}=f_{i, j}(z)$. To motivate the solution from the other direction: if we already know that we have to consider a matrix put together from the $f_{i, j}(z)$ power series, then splitting up the matrix by the powers of $z$ we obtain that $\sum_{n=0}^{\infty} A^{n} \cdot z^{n}$ encodes the generating functions. Now, $\sum_{n=0}^{\infty} A^{n} \cdot z^{n}$ can be calculated using the usual argument about geometric power series. That is

$$
\sum_{n=0}^{\infty} A^{n} \cdot z^{n}=\sum_{n=0}^{\infty}(A \cdot z)^{n}=\frac{1}{1-A z}=(1-A z)^{-1}=(I-A z)^{-1}
$$




$$
\text { "tmcs-horvath" — 2013/5/26 - 0:05 — page } 7 \text { - \#5 }
$$

using that the sum of a geometric series is $\sum x^{n}=1 /(1-x)$. The entries of the inverse matrix are the desired generating functions, each being a rational function of $z$.

Now, these arguments should be made precise in order to satisfy the needs of not only a physicist but a mathematician, as well. One way would be to define the power series ring $R[[z]]$ over an arbitrary (not necessarily commutative) ring $R$ (see e.g. $[4,11]$ ). Then one could prove that there exists a natural isomorphism between the ring $\mathbb{R}^{n \times n}[[z]]$ (the ring of power series over the $n$ by $n$ matrices) and the ring $\mathbb{R}[[z]]^{n \times n}$ (the ring of $n$ by $n$ matrices of power series). After this, proving that every step of (1) holds comes down to simply calculating that the equations

$$
(I-A z) \cdot\left(\sum_{n=0}^{\infty} A^{n} \cdot z^{n}\right)=I=\left(\sum_{n=0}^{\infty} A^{n} \cdot z^{n}\right) \cdot(I-A z)
$$

hold in the power series ring $\mathbb{R}^{n \times n}[[z]]$.

Even though the argument on formal power series is mathematically correct and can be applied here, proving that the steps of (1) are valid is usually achieved using analytical methods. That is, one argues that the series in (1) is convergent someplace and that the sum is really $(I-A z)^{-1}$. Let $\mu$ be the eigenvalue of $A$ having the highest absolute value. From the theory of functional operations and linear algebra it follows that the series $\sum_{n=0}^{\infty}(A \cdot z)^{n}$ is convergent if $|z|<1 /|\mu|$. Then the standard arguments

$$
\begin{aligned}
\sum_{n=0}^{\infty} A^{n} \cdot z^{n}=\lim _{k \rightarrow \infty} \sum_{n=0}^{k} A^{n} & \cdot z^{n}=\lim _{k \rightarrow \infty} \sum_{n=0}^{k}(A z)^{n} \\
& =\lim _{k \rightarrow \infty}\left(I-(A z)^{k+1}\right) \cdot(I-A z)^{-1}=(I-A z)^{-1}
\end{aligned}
$$

give us the formula needed.

Finally, the last exercise is to calculate the generating function for $\omega_{n}(1 \rightarrow 3)$. This is simply calculating the inverse of the matrix $(I-A z)$ by e.g. Cramer's rule, and then take the entry in the first row and third column. This entry is a rational function, the corresponding generating function is the Taylor series of this rational function.

\subsection{The solution for a student in mathematics}

At ELTE discrete mathematics is taught on two levels, standard and advanced. The advanced course covers adjacency matrices and their properties, the 
standard course does not. At DE and at SzTE the class called 'Combinatorics' covers adjacency matrices and their properties. Nevertheless, the notion of adjacency matrix can easily be explained even for high school students (see e.g. the third of this series of papers [7]): present the matrix $A$, where $[A]_{i}^{j}$ is the number of edges from vertex $i$ to vertex $j$.

Now, let $\omega_{n}(i \rightarrow j)$ denote the number of $n$ step walks from vertex $i$ to vertex $j$. Note, that the number of 1 step walks from vertex $i$ to $j$ is the element $[A]_{i}^{j}$, that is $[A]_{i}^{j}=\omega_{1}(i \rightarrow j)$. It can be proved by mathematical induction that $A^{n}$ encodes the number of $n$ step walks from a point to another. Indeed, an $n+1$ step walk from $i$ to $j$ consists of an $n$ step walk from $i$ to $k$ (for some vertex $k$ ) and then a 1 step walk from $k$ to $j$. To compute the number of $n+1$ step walks, we need to add the product of these two numbers for all vertices $k$. That is $\omega_{n+1}(i \rightarrow j)=\sum_{k=1}^{n} \omega_{n}(i \rightarrow k) \omega_{1}(k \rightarrow j)$. By the induction hypothesis $\omega_{n}(i \rightarrow k)$ is the $i k$ entry of the matrix $A^{n}$, and $\omega_{1}(k \rightarrow j)=[A]_{k}^{j}$, thus $\omega_{n+1}(i \rightarrow j)=\sum_{k=1}^{n} \omega_{n}(i \rightarrow k) \omega_{1}(k \rightarrow j)$ is the $i j$ entry of $A^{n+1}$ by matrix multiplication. For the particular graph $G$, the matrix giving the number of 3 step walks is $A^{3}$, which can be easily verified to be the same matrix as to what Will wrote on the board. This argument is understandable for any student who finished an introductory linear algebra course (2nd semester at ELTE and DE, 1st semester at SzTE).

The third exercise was to provide the generating function for walks from $i$ to $j$. The concept of the generating function can be explained to students not familiar with the usual theory of power series and analytic functions. This is the way, for example in [4], where it is a formal, infinite expression, and is taught at ELTE in the 2nd semester, at DE in the 1st semester and at SzTE in the 3rd semester. In this case, however, one would need power series over a noncommutative ring (namely, over the matrix ring $\mathbb{R}^{n \times n}$ ), which is not discussed at any undergraduate course at any of the three universities. Thus we base our theory of generating functions on analysis. The generating function is an analytic function defined by its power series $\sum_{n=0}^{\infty} \omega_{n}(i \rightarrow j) \cdot z^{n}$, that is the coefficient of $z^{n}$ is the number of $n$ step walks from $i$ to $j$. The theory of generating functions uses only the theory of power series from analysis, and therefore it can be explained to any student having that knowledge.

For notions and theorems in calculus we refer to [9], for the generating function method and its uses in Combinatorics can be found in [1]. We have already observed via solving the previous exercise that $\omega_{n}(i \rightarrow j)$ is the $i j$ entry of the matrix $A^{n}$. As the problem asks to find all generating functions at the same 


$$
\text { "tmcs-horvath" — 2013/5/26 - 0:05 — page } 9-\# 7
$$

time, it might suggest to arrange them in a matrix. Then, it does not take much of a leap to consider the matrix power series $\sum_{n=0}^{\infty} A^{n} \cdot z^{n}$. Nevertheless, the theory of matrix power series is not part of the analysis classes, hence needs some explanation.

How can one define the convergence of a matrix-series? The closest notion is the convergence in normed vector spaces, e.g. in $\mathbb{R}^{n}$. This is covered at ELTE in Analysis 3, at DE and at SzTE in Multiple variable analysis. If we consider the $n$ by $n$ matrices simply as an $n^{2}$ dimensional vector space, then it becomes a normed space, for example, with the standard Euclidean distance. Being finite dimensional, any two norms are equivalent over this space. Hence, we are allowed to use the pointwise convergence saying that a series of matrices is convergent if and only if each entry involves a convergent series in the usual sense in $\mathbb{R}$. Hence, for our purposes it is enough to consider this matrix power series as putting the $n^{2}$-many usual power series in a table of $n$ rows and $n$ columns. Moreover, by the above arguments we can proceed with all computations formally using the usual matrix addition and multiplication. Thus, every generating function $f_{i, j}(z)$ is convergent (in some neighborhood of 0 ) if and only if the matrix series $\sum_{n=0}^{\infty} A^{n} \cdot z^{n}$ is convergent (in some neighborhood of 0 ). We investigate the latter one. By the usual argument about geometric power series, we have

$$
\begin{aligned}
\sum_{n=0}^{\infty} A^{n} \cdot z^{n}=\lim _{k \rightarrow \infty} \sum_{n=0}^{k} & A^{n} \cdot z^{n}=\lim _{k \rightarrow \infty} \sum_{n=0}^{k}(A z)^{n} \\
& =\lim _{k \rightarrow \infty}\left(I-(A z)^{k+1}\right) \cdot(I-A z)^{-1}=(I-A z)^{-1}
\end{aligned}
$$

One still has to make sure that every equation of (2) holds, and every step makes sense. That is, one needs to determine the set of numbers $z$ for which $\lim _{k \rightarrow \infty}\left(I-(A z)^{k+1}\right)=I$, and for which the matrix $I-A z$ is invertible. The latter question can be decided easily: $I-A z$ is invertible if and only if $z \lambda \neq 1$ for any eigenvalue $\lambda$ of $A$. Let $\mu$ be the eigenvalue of $A$ having the highest absolute value. Then $I-A z$ is invertible for $|z|<1 /|\mu|$.

Now, consider the $\operatorname{limit}_{k \rightarrow \infty}(A z)^{k}$. Let $J$ be the normal form of $A$, that is there exists an invertible matrix $Q$ such that $J=Q^{-1} A Q$ consists of only Jordan blocks. Then $A=Q J Q^{-1}$ and thus $(A z)^{k}=Q J^{k} Q^{-1} z^{k}$. If $A$ is an $n$ by $n$ matrix, then the entries of $J^{k}$ are bounded by $k^{n}|\mu|^{k}$. In fact, as $A$ is symmetric, $J$ is diagonal and thus the entries of $J^{k}$ are bounded by $|\mu|^{k}$. The elements of $Q$ and $Q^{-1}$ are constants. Thus $\lim _{k \rightarrow \infty}(A z)^{k}=\lim _{k \rightarrow \infty} Q J^{k} Q^{-1} z^{k}=0$ for $|z|<1 /|\mu|$, and hence $\lim _{k \rightarrow \infty}\left(I-(A z)^{k+1}\right)=I$ in the same neighborhood. 
This technique is taught at ELTE in Algebra 2, at DE in Linear algebra 2, at SzTE in Linear algebra.

Finally, we need to compute the inverse of $I-A z=I-z A$. The usual Gaussian elimination method is of little help here, as $z$ is a variable in the entries of $I-z A$, and it is hard to compute with it. But the inverse of a matrix can be calculated by Cramer's rule, using the adjugate matrix, which is taught in any introductory linear algebra course (Algebra 2 at ELTE, Linear algebra 1 at DE, Linear algebra at SzTE). For a matrix $M$, let $M_{i j}$ denote the matrix obtained from $M$ by omitting the $i$ th column and $j$ th row. Then the adjugate matrix of $M$ is the matrix $N$ whose $i j$ entry is $(-1)^{i+j} \operatorname{det} M_{i j}$. By Cramer's rule if $M$ is invertible, then $M^{-1}=N / \operatorname{det} M$. That is, the $i j$ entry of $M^{-1}$ is $(-1)^{i+j} \operatorname{det} M_{i j} / \operatorname{det} M$. Applying it to the third exercise in the movie for the matrix $M=I-z A$, we obtain that the generating function of walks from $i$ to $j$ is the fraction $(-1)^{i+j} \operatorname{det}\left(I_{i j}-z A_{i j}\right) / \operatorname{det}(I-z A)$. This is almost the same as Will's solution, except for the $(-1)^{i+j}$ factor at the beginning. Sometimes the notation $\operatorname{det}\left(I_{i j}-z A_{i j}\right)$ covers the $(-1)^{i+j}$ factor by itself, but it may as well be an oversight from the creators of the movie. Another difference is that Will denoted the identity matrix by $\mathbf{1}$ rather than by $I$.

In the fourth exercise, we are to determine the generating function for walks from 1 to 3 . Having obtained the general formula in the previous step of the problem, it is not hard to substitute $i=1$ and $j=3$ :

$$
\begin{aligned}
& \sum_{n=0}^{\infty} \omega_{n}(1 \rightarrow 3) z^{n}=(-1)^{1+3} \operatorname{det}\left(I_{13}-z A_{13}\right) / \operatorname{det}(I-z A) \\
& \quad=\left|\begin{array}{ccc}
-z & 0 & -z \\
1 & -2 z & -z \\
-z & 0 & 1
\end{array}\right| \cdot\left|\begin{array}{cccc}
1 & -z & 0 & -z \\
-z & 1 & -2 z & -z \\
0 & -2 z & 1 & 0 \\
-z & -z & 0 & 1
\end{array}\right|^{-1}=\frac{2 z^{3}+2 z^{2}}{4 z^{4}-2 z^{3}-7 z^{2}+1}
\end{aligned}
$$

Any student finished an introductory linear algebra course (Algebra 2 at ELTE, Linear algebra 1 at DE, Linear algebra at SzTE) should not have a problem to arrive at this formula after some guidance from the teacher. The personal experience of the second author is that the students do not usually find the formula on their own, but deduce it with no problems after they know Cramer's rule should be applied. Here, -1 is a root of both the nominator and the denominator, hence 
we can reduce the fraction by $z+1$ :

$$
\begin{aligned}
\sum_{n=0}^{\infty} \omega_{n}(1 \rightarrow 3) z^{n}=\frac{2 z^{3}+2 z^{2}}{4 z^{4}-2 z^{3}-7 z^{2}+1} & \\
& =\frac{(z+1) 2 z^{2}}{(z+1)\left(4 z^{3}-6 z^{2}-z+1\right)}=\frac{2 z^{2}}{4 z^{3}-6 z^{2}-z+1}
\end{aligned}
$$

To obtain the coefficients of the power series, we need to compute the Taylor series of this function. Taylor series are covered by Analysis 2 at ELTE, Differentiation at DE and at SzTE. We apply the well known formula from calculus $f(z)=$ $\sum_{n=0}^{\infty} \frac{f^{(n)}(0)}{n !} z^{n}$, where $f^{(n)}(0)$ is the $n$th derivative of $f$ at 0 and $n !=1 \cdot 2 \cdots \cdots n$ is the product of positive integers from 1 to $n$ (and $0 !=1$ by definition). Here, $f(z)=2 z^{2} /\left(4 z^{3}-6 z^{2}-z+1\right)$, thus we need to determine its derivatives at 0 . Let $h(z)=2 z^{2}, g(z)=4 z^{3}-6 z^{2}-z+1$, then $f(z)=h(z) / g(z)$. Will gave the coefficients of the first six terms, which can be obtained by computing the first six derivatives of $f$. As $f$ is a fraction of two polynomials, it can be quite tedious to do the derivations by hand. Therefore we are going to use an easy trick to reduce the computational time. As $h / g=f$, we have $h=f g$ and $h^{(k)}=(f g)^{(k)}$. It is easy to determine the derivatives by inductively applying the product rule for derivatives. Now, $h^{\prime}(z)=4 z, h^{\prime \prime}(z)=4, h^{\prime \prime \prime}(z)=0, g^{\prime}(z)=12 z^{2}-12 z-1, g^{\prime \prime}(z)=24 z-12$, $g^{\prime \prime \prime}(z)=24, g^{(4)}(z)=0$. Then we have $h(0)=h^{\prime}(0)=h^{\prime \prime \prime}(0)=0, h^{\prime \prime}(0)=4$, $g(0)=1, g^{\prime}(0)=-1, g^{\prime \prime}(0)=-12, g^{\prime \prime \prime}(0)=24$. We obtain a system of linear equations to solve for the derivatives of $f$ in 0 :

$$
\begin{array}{rlrl}
h(0)= & f(0) g(0) & & \Rightarrow f(0)=0, \\
h^{(1)}(0)= & f^{(1)}(0) g(0)+f(0) g^{(1)}(0) & & \Rightarrow f^{(1)}(0)=0, \\
h^{(2)}(0)= & f^{(2)}(0) g(0)+2 f^{(1)}(0) g^{(1)}(0)+f(0) g^{(2)}(0) & & \Rightarrow f^{(2)}(0)=4, \\
h^{(3)}(0)= & f^{(3)}(0) g(0)+3 f^{(2)}(0) g^{(1)}(0) & & \\
& +3 f^{(1)}(0) g^{(2)}(0)+f(0) g^{(3)}(0) & & \\
h^{(4)}(0)= & f^{(4)}(0) g(0)+4 f^{(3)}(0) g^{(1)}(0)+6 f^{(2)}(0) g^{(2)}(0)=12, \\
& +4 f^{(1)}(0) g^{(3)}(0)+f(0) g^{(4)}(0) & & \\
h^{(5)}(0)= & f^{(5)}(0) g(0)+5 f^{(4)}(0) g^{(1)}(0)+10 f^{(3)}(0) g^{(2)}(0) & & \\
& +10 f^{(2)}(0) g^{(3)}(0)+5 f^{(1)}(0) g^{(4)}(0)+f(0) g^{(5)}(0) & & \Rightarrow f^{(5)}(0)=2160, \\
h^{(6)}(0)= & f^{(6)}(0) g(0)+6 f^{(5)}(0) g^{(1)}(0)+15 f^{(4)}(0) g^{(2)}(0) & & \\
& +21 f^{(3)}(0) g^{(3)}(0)+15 f^{(2)}(0) g^{(4)}(0) & & \\
& +6 f^{(1)}(0) g^{(5)}(0)+f(0) g^{(6)}(0) & & \Rightarrow f^{(6)}(0)=67680 .
\end{array}
$$




$$
\text { "tmcs-horvath" — 2013/5/26 - 0:05 — page } 12 \text { - \#10 }
$$

After dividing these numbers by the appropriate factorials we obtain

$$
\sum_{n=0}^{\infty} \omega_{n}(1 \rightarrow 3) z^{n}=2 z^{2}+2 z^{3}+14 z^{4}+18 z^{5}+94 z^{6}+\ldots
$$

which is, again, what Will wrote on the board.

We finish the section by summarizing the required courses at each university for the four exercises in Table 1.

Table 1. Required classes for the exercises on the board

\begin{tabular}{|l|l|l|l|}
\hline ex. & ELTE (semester) & DE (semester) & SzTE (semester) \\
\hline$(1)$ & Discr. math. 1 (1) & Combinatorics (1) & Combinatorics (3) \\
\hline$(2)$ & Algebra 2 (2) & Linear algebra 1 (2) & Linear algebra (1) \\
\hline \multirow{3}{*}{$(3)$} & Discr. math. 2 (2) & Combinatorics (1) & Combinatorics (3) \\
& Algebra 2 (2) & Linear algebra 2 (3) & Linear algebra (1) \\
& Analysis 3 (3) & Mult. var. analysis (4) & Mult. var. analysis (3) \\
\hline$(4)$ & Algebra 2 (2) & Linear Algebra 1 (2) & Linear algebra (1) \\
& Analysis 2 (2) & Differentiation (3) & Differentiation (2) \\
\hline
\end{tabular}

\section{Eigenvalues, eigenvectors}

When Gerald Lambeau comes into the main lecture hall full of students hoping to reveal the identity of the mystery person solving the first problem, we see in the background that two problems are written and solved on the main board. Both of these problems come from linear algebra, they are about determining eigenvalues and eigenvectors of a particular matrix. These are taught in the course Algebra 2 (semester 2) at ELTE, in Linear algebra 2 (semester 3) at DE and in Linear algebra (semester 1) at SzTE.

It is explained on the left hand side of the board how the eigenvalues of the matrix

$$
A=\left(\begin{array}{ccc}
1 & 1 & 0 \\
1 & 1 & -2 \\
2 & 1 & 0
\end{array}\right)
$$

should be computed. This matrix has one real (not rational) and two complex eigenvalues. One can obtain these eigenvalues by solving a third degree equation. Since none of these eigenvalues are nice, this particular exercise may be more 


$$
\text { "tmcs-horvath" — 2013/5/26 - 0:05 — page } 13 \text { — \#11 }
$$

suitable to explain Cardano's formula on the solution of a third degree equation, which is taught in the course Algebra 1 (semester 1) at ELTE, in Introduction to algebra and number theory (semester 2) at DE and in Classical algebra (semester 2) at SzTE.

The second problem written on the board of the main lecture hall is again an eigenvalue problem for the matrix

$$
A=\left(\begin{array}{ccc}
2 k & -k & -k \\
k & 2 k & -k \\
k & k & 2 k
\end{array}\right)
$$

In the movie, above this matrix one can see on the board that 0 and $3 k$ are two eigenvalues, and $3 k$ is an eigenvalue with multiplicity two (called degenerate eigenvalue on the board). We can read the particular eigenvectors corresponding to these eigenvalues, as well. However, neither 0 nor $3 k$ are eigenvalues of the matrix $A$ (except in the case $k=0$ ). Therefore we believe, that the creators of the movie may have made a mistake here and wanted to put the symmetric matrix

$$
B=\left(\begin{array}{ccc}
2 k & -k & -k \\
-k & 2 k & -k \\
-k & -k & 2 k
\end{array}\right)
$$

on the board instead. One can easily determine that the three eigenvalues of $B$ are $0,3 k, 3 k$. The eigenvector corresponding to the eigenvalue 0 is

$$
\left(\begin{array}{l}
1 \\
1 \\
1
\end{array}\right)
$$

and the two-dimensional eigenspace is generated by the vectors

$$
\left(\begin{array}{c}
1 \\
-1 \\
0
\end{array}\right),\left(\begin{array}{c}
1 \\
0 \\
-1
\end{array}\right) \text {. }
$$

\section{Trees}

The second problem on the hallway board was written specifically to challenge the person who solved the first problem. It consists of two exercises: 


$$
\text { "tmcs-horvath" — 2013/5/26 - 0:05 — page } 14 \text { - \#12 }
$$

Problem 2. (1) How many trees are there with $n$ labeled vertices?

(2) Draw all the homeomorphically irreducible trees with $n=10$.

Will gives the answer $n^{n-2}$ to the first question and draws eight graphs to answer the second question.

The result of the first question is known as Cayley's formula [3] but has been discovered first in 1860 by Borchardt [2]. There are several different ways to prove it, maybe one of the most well-known proofs is the one due to Prüfer [10], assigning a so-called Prüfer code to every tree. Cayley's theorem with this proof is taught in Discrete mathematics 1 (semester 1) at ELTE, in Combinatorics (semester 1) at DE and in Combinatorics (semester 3) at SzTE.

Now, we move on to the second problem about homeomorphically irreducible trees. A tree is homeomorphically irreducible (or sometimes called series-reduced) if it has no vertex of degree two. The first results about homeomorphically irreducible trees are due to Harary and Prins [5]. For example they list all such trees having at most 12 vertices, and in particular list those having exactly 10 vertices. Even though homeomorphically irreducible trees are not part of any undergraduate course at any of the three universities, by using elementary graph theoretical results, one can find all homeomorphically irreducible trees having 10 vertices. It is interesting that the creators' of the movie made a mistake: Will draws 8 trees on the board, while there exist 10 homeomorphically irreducible trees with 10 vertices.

Let us label the vertices by $1, \ldots, 10$, their degrees by $d_{1}, \ldots, d_{10}$. Assume that the degrees are in decreasing order. Now, the sum of the degrees is 18 as the tree has 9 edges. If there are $l$ leaves and $10-l$ non-leaves, then the sum of the degrees $(18)$ is at least $l+3 \cdot(10-l)=30-2 l$, thus $l \geq 6$. If there are 9 leaves and 1 non-leaf, then we obtain the star: 1 is connected to every other vertex. If there are 8 leaves then $d_{1}+d_{2}=10$, and $d_{1} \geq d_{2} \geq 3$. Thus $d_{1}=7$ and $d_{2}=3$, or $d_{1}=6$ and $d_{2}=4$, or $d_{1}=d_{2}=5$. All three of them gives one homeomorphically irreducible tree, where 1 and 2 are connected and the leaves are connected to them according to their degrees. If there are 7 leaves, then $d_{1}+d_{2}+d_{3}=11$, and $d_{1} \geq d_{2} \geq d_{3} \geq 3$. Thus either $d_{1}=d_{2}=4$ and $d_{3}=3$, or $d_{1}=5$ and $d_{2}=d_{3}=3$. The first case produces two trees: one where the two degree 4 nodes are connected and one where they are not connected. The second case produces two trees, as well: one where the two degree 3 nodes are not connected and one where they are connected. The latter tree is missing from the board in the movie, which must be the creators' mistake. Finally, if there are 6 leaves, 
then $d_{1}+d_{2}+d_{3}+d_{4}=12, d_{1} \geq d_{2} \geq d_{3} \geq d_{4} \geq 3$, hence $d_{1}=d_{2}=d_{3}=d_{4}=3$. This produces two more trees: one where there exists a degree 3 node with no leaves attached to it, and one where no such node exists. This latter graph is, again, missing from the board, which is another mistake in the movie. Altogether there are 10 (rather than 8) homeomorphically irreducible trees with 10 nodes (see Figures 2 and 3). If the reader is further interested in homeomorphically irreducible trees, they can consult e.g. [1].

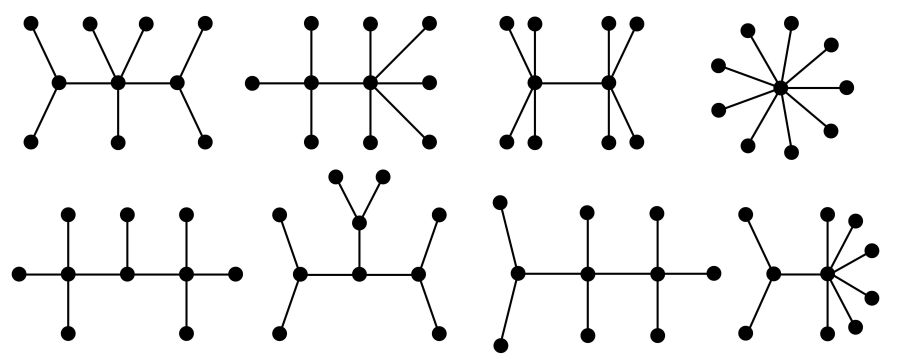

Figure 2. The 8 homeomorphically irreducible trees on the board

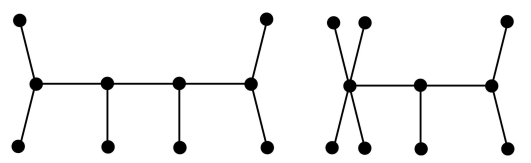

Figure 3. The 2 missing homeomorphically irreducible trees from the board

\section{Chromatic polynomial}

The problem we see being solved by Gerald Lambeau and Will Hunting together is to determine the chromatic polynomial of the 3-Sun graph, i.e. of the graph having vertices $a, b, c, d, e, f$ and edges $a b, a c, a d, a e, b c, b d, b f, c e, c f$. This is a planar graph which looks like a triangle with connecting the midpoints of the sides (Figure 4).

The chromatic polynomial of a graph $G$ is a function $p_{G}(k)$, which gives the number of well-colorings of $G$ by $k$ different colors. A coloring of the vertices is called a well-coloring if adjacent vertices are colored by a different color. It is 


$$
\text { "tmcs-horvath" — 2013/5/26 - 0:05 — page } 16 \text { - \#14 }
$$

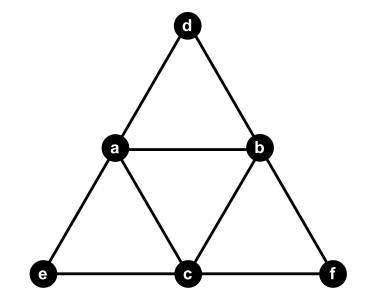

Figure 4. The 3-Sun graph

a computationally hard problem to determine the smallest number of colors for which there exists a well-coloring of a graph with that many colors. Obviously the chromatic number is the smallest positive integer which is not the root of the chromatic polynomial. Thus it is computationally hard to determine the chromatic polynomial for a graph in general, as well. For particular graphs it can be easy to determine this polynomial, e.g. the chromatic polynomial of the complete graph having $n$ vertices is $k(k-1) \ldots(k-n+1)$ or the chromatic polynomial for the empty graph is simply $k^{n}$.

The chromatic number is taught at every introductory discrete mathematics or combinatorics course. That is, it is covered in discrete mathematics 1 (semester 1) at ELTE, in Combinatorics (semester 1) at DE and in Combinatorics (semester 3) at SzTE. Although the chromatic polynomial can be introduced in an elementary way, it is only part of the course taught at DE. In the following we introduce how one could build up a lecture on this exercise.

It is already interesting that this particular function $p_{G}(k)$ is indeed a polynomial. Its degree is at most $n$ if $G$ has $n$ vertices. It can be shown as follows. A well-coloring creates a partition of the vertices of the graph by the color classes, and each color class is an independent set in the graph. As $G$ is finite, there are finitely many ways to partition it as the union of independent sets. Thus the number of colorings can be computed by calculating the number of colorings giving a particular partitioning and then summing these numbers for all possible partitions. Let $P$ be a partitioning with $d$ independent sets. Then the first independent set can be colored by $k$ colors, the next set by $(k-1)$ colors, etc. The last independent set can be colored by $(k-d+1)$ colors, and thus the number of colorings by $k$ colors determining the partition $P$ is $k(k-1) \ldots(k-d+1)$. This is a degree $d$ polynomial in $k$, where $d \leq n$.

Now, we determine the chromatic polynomial of the Sun graph $G$ in the movie. Recall that the graph $G$ consists of vertices $a, b, c, d, e, f$ and edges $a b, a c, a d$, 
$a e, b c, b d, b f, c e, c f$ (see Figure 4). Let us count the number of well-colorings by $k$ colors. We can color the vertex $a$ by $k$ colors, which leaves $(k-1)$ colors to choose from to color the vertex $b$. Then, we will have $(k-2)$ colors to choose from to color the vertex $c$. Finally, the remaining three vertices $(d, e$ and $f)$ can all be colored independently by $(k-2)$ colors, as they have only two neighbors which are colored already by two different colors. Thus $p_{G}(k)=k(k-1)(k-2)^{4}$, as Will Hunting and Gerald Lambeau deduced together. Nevertheless, they obtained this result in a different way. They may have obtained this result from the following lemma.

LEMmA 1. If $G$ and $H$ are two graphs intersecting in a complete graph then

$$
p_{G \cup H}(k)=\frac{p_{G}(k) p_{H}(k)}{p_{G \cap H}(k)} .
$$

Proof. Let us color first $G \cap H$. Now, $G \cap H$ is a complete graph, thus all its vertices must be of different color. Fix a well-coloring of $G \cap H$, and let $q_{G}(k)$ be the number of well-colorings of $G$ extending this particular coloring of $G \cap H$. Observe, that for another well-coloring of $G \cap H$, it can be extended in $q_{G}(k)$-many ways into a well-coloring of $G$, as well. Thus we have $p_{G}(k)=$ $p_{G \cap H}(k) \cdot q_{G}(k)$. Similarly, let $q_{H}(k)$ be the number of well-colorings of $H$ extending a fixed coloring of $G \cap H$. Then $p_{H}(k)=p_{G \cap H}(k) \cdot q_{H}(k)$. Now, $p_{G \cup H}(k)$ can be calculated by counting how many ways can a well-coloring of $G \cap H$ be extended into a well-coloring of $G \cup H$. As $G \backslash(G \cap H)$ and $H \backslash(G \cap H)$ are independent, we have

$$
\begin{aligned}
p_{G \cup H}(k)=p_{G \cap H}(k) \cdot q_{G}(k) \cdot q_{H}(k) & \\
= & \frac{p_{G \cap H}(k) \cdot q_{G}(k) \cdot p_{G \cap H}(k) \cdot q_{H}(k)}{p_{G \cap H}(k)}=\frac{p_{G}(k) \cdot p_{H}(k)}{p_{G \cap H}(k)} .
\end{aligned}
$$

By induction on the number of graphs, one can immediately prove

Corollary 2. If $G_{1}, G_{2}, \ldots, G_{d}$ are graphs such that any two intersects in the very same complete graph, then

$$
p_{G_{1} \cup G_{2} \cup \cdots \cup G_{d}}(k)=\frac{p_{G_{1}}(k) p_{G_{2}}(k) \ldots p_{G_{d}}(k)}{p_{G_{1} \cap G_{2} \cap \cdots \cap G_{d}}(k)^{d-1}} .
$$




$$
\text { "tmcs-horvath" — 2013/5/26 - 0:05 — page } 18 \text { - \#16 }
$$

Now choose $G_{1}$ to be the subgraph spanned by the vertices $a, b, c, d, G_{2}$ to be the subgraph spanned by the vertices $a, b, c, e$, and $G_{3}$ to be the subgraph spanned by the vertices $a, b, c, f$. Consider the graph $G_{1}$. Here, the vertex $a$ can be colored by $k$ colors, the vertex $b$ can be colored by $k-1$ colors, and $c$ and $d$ can be colored independently by any of the remaining $k-2$ colors. The same reasoning works for the graphs $G_{2}$ and $G_{3}$. Thus $p_{G_{i}}(k)=k(k-1)(k-2)^{2}$. The chromatic polynomial of the complete graphs of three vertices is $k(k-1)(k-2)$, and thus by Corollary 2 we have

$$
p_{G}(k)=p_{G_{1} \cup G_{2} \cup G_{3}}(k)=\frac{p_{G_{1}}(k) p_{G_{2}}(k) p_{G_{3}}(k)}{p_{G_{1} \cap G_{2} \cap G_{3}}(k)^{2}}=\frac{k^{3}(k-1)^{3}(k-2)^{6}}{k^{2}(k-1)^{2}(k-2)^{2}} .
$$

This formula is now exactly the same as in the movie. For further reading on chromatic polynomials we suggest the reader to take a look at [8, ex. 9.36-9.49].

\section{References}

[1] F. Bergeron, G. Labelle and P. Leroux., Combinatorial species and tree-like structures, Vol. 67, Encyclopedia of Mathematics and its Applications, Cambridge University Press, Cambridge, 1998, Translated from the 1994 French original by Margaret Readdy, With a foreword by Gian-Carlo Rota.

[2] C. W. Borchardt, Ueber eine der Interpolation entsprechende Darstellung der Eliminations-Resultante, J. Reine Angew. Math. 57 (1860), 111-121.

[3] A. Cayley, A theorem on trees, Quaterly Journal of Pure and Applied Mathematics XXIII (1889), 376-378.

[4] P. Hajnal, Összeszámlálási problémák, Polygon, Szeged, 1997.

[5] F. Harary and G. Prins, The number of homeomorphically irreducible trees, and other species, Acta Math. 101 (1959), 141-162.

[6] J. Korándi and G. Pluhár, Mathematics and Good Will Hunting I, Teach. Math. Comput. Sci. 10, no. 2 (2012), 375-388.

[7] J. Korándi and G. Pluhár, Mathematics and Good Will Hunting III, Teach. Math. Comput. Sci. (2013), submitted.

[8] L. Lovász, Combinatorial problems and exercises, AMS Chelsea Publishing, second edition, Providence, RI, 2007.

[9] G. Pólya and G. Szegö, Problems and theorems in analysis, Vol. I: Series, integral calculus, theory of functions, Springer-Verlag, New York, 1972, Translated from the German by D. Aeppli, Die Grundlehren der mathematischen Wissenschaften, Band 193.

[10] H. Prüfer, Neuer Beweis eines Satzes über Permutationen, Arch. d. Math. u. Phys. (3) 27 (1918), 142-144. 


$$
\text { "tmcs-horvath" — 2013/5/26 - 0:05 — page } 19-\# 17
$$

[11] R. P. Stanley, Enumerative combinatorics Vol. 2, Vol. 62 of Cambridge Studies in Advanced Mathematics, Cambridge University Press, Cambridge, 1999, with a foreword by Gian-Carlo Rota and appendix 1 by Sergey Fomin.

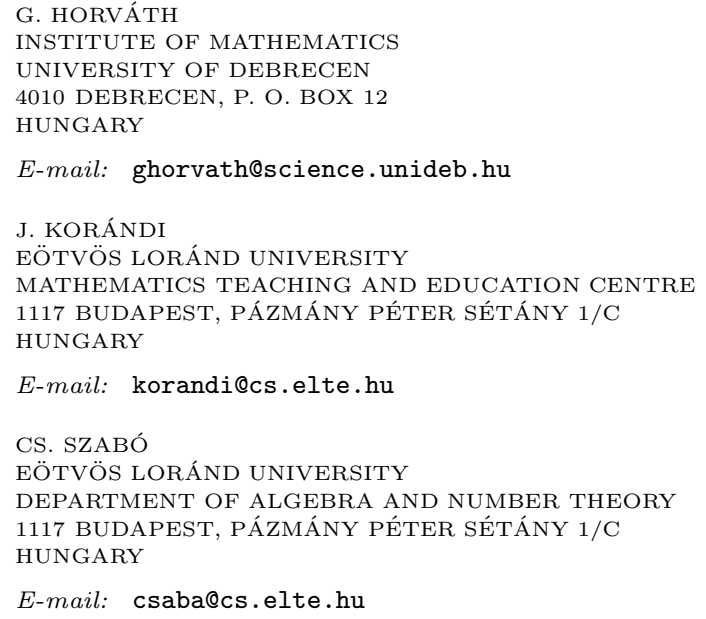

(Received February, 2012) 